\title{
Cost optimization of thermoelectric materials for power generation: The case for $Z T$ at (almost) any cost
}

C. Dames ${ }^{1,2}$

${ }^{1}$ Mechanical Engineering, University of California, Berkeley.

${ }^{2}$ Materials Sciences Division, Lawrence Berkeley National Laboratory.

cdames@berkeley.edu

\begin{abstract}
We quantify the tradeoff between $Z T$ and materials cost for thermoelectric energy scavengers, considering 31 real materials. It is nearly always practical to aggressively miniaturize the legs until the overall system cost is limited by the heat exchangers and ceramic spreader plates. For thermoelectric materials costing less than $C_{c r i t .}^{\prime \prime \prime} \sim 1 \$ \mathrm{~cm}^{3}(\sim 100 \$ / \mathrm{kg}$ ), the material might as well be free, and even costs as high as $\sim 10$ $\$ / \mathrm{cm}^{3}(\sim 1,000 \$ / \mathrm{kg})$ may still be acceptable. Thus, the materials goal is maximizing ZT at (almost) any cost.
\end{abstract}

\section{Keywords}

thermoelectric; cost; optimization; system 


\section{Introduction}

This Viewpoint paper is concerned with identifying the best thermoelectric materials for cost-effective energy scavenging: systems that generate the most electrical power for the least capital investment. We denote this system-level metric as $G$, which carries the dimensions of [cost/power], here expressed as $[\$ / \mathrm{W}]$. This is closely related to the levelized cost of electricity (LCOE, typically expressed as [cents/kW-hr]), which spreads the capital costs out over the life of the project $[1,3]$.

Traditionally thermoelectric materials have been ranked by their dimensionless figures of merit, ZT, which has been known for more than a century $[4,5]$ to determine the efficiency of a thermoelectric generator with prescribed junction temperatures. To incorporate economics one must consider the essential tradeoff depicted in Fig. 1: the competition between high ZT and low material cost. Indeed, this tradeoff frames two major themes of contemporary thermoelectric materials research:

-The nanostructuring approach: How much of a premium should we be willing to pay for increased ZT?

- The polymer approach: How much ZT can we afford to sacrifice when using a material that is orders of magnitude less expensive?

A third and intimately related question is:

-The limit of free material: How cheap is cheap enough? What is the critical material cost, $C_{\text {Crit. }}^{\prime \prime}$, below which the material might as well be free? Clearly the system cost must remain finite, due to the heat exchanger (HX) and ceramic plate heat spreaders.

Addressing these three questions requires analyzing the complete energy scavenging system, including both the heat exchanger (and associated heat spreading plates) and the thermoelectric material. Furthermore, a suitable analysis must couple the thermal, electrical, and cost aspects, to capture the tradeoffs depicted schematically in Fig. 1. Cost issues in thermoelectric materials selection have been considered previously [1, 6-10]; as summarized in Ref. [6] this includes one-way [8, 9] and later two-way

Fig. 1. The tradeoffbetween $Z T$ and material cost when optimizing a thermoelectric system for minimal $\$ / W$. The "nanostructuring approach" invests in premium materials with increased $Z T$, while the "polymer approach" sacrifices some $Z T$ to use much less expensive materials. Either approach must be done wisely: compare the strategies marked $\checkmark$ versus $x$. Below some $C_{C r i t}^{\prime \prime}$, the material costs are so low as to be practically free; in this regime system optimization calls for pursuing high $Z T$ at any cost. This plot is made quantitative below in Figs. 4 and 5.

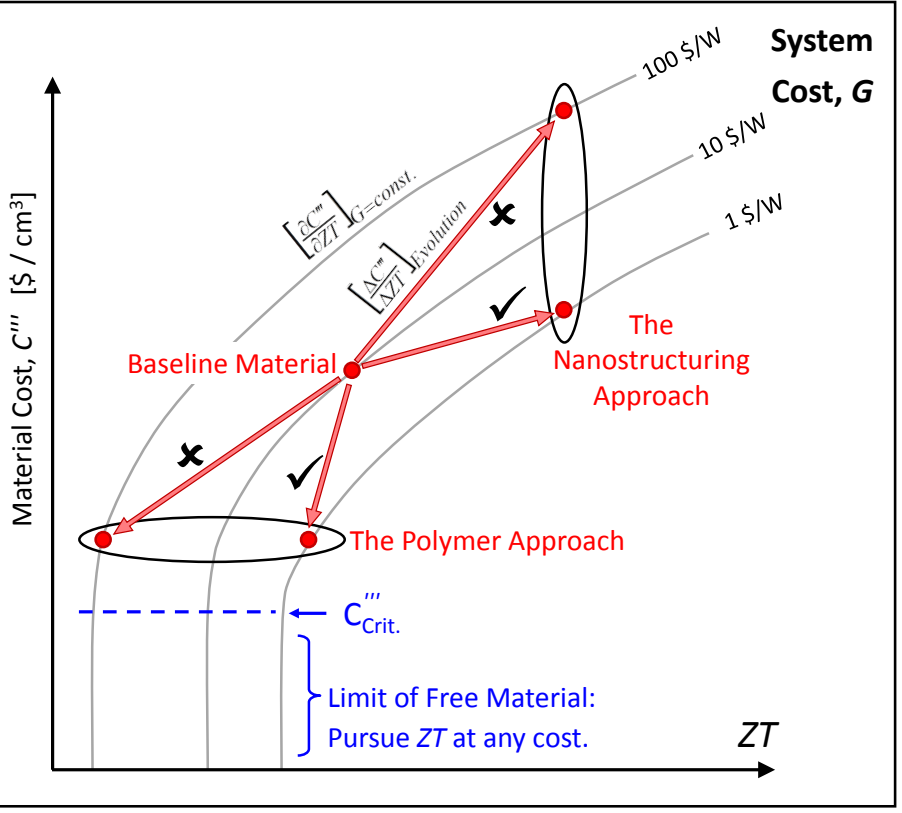


$[1,6,10]$ coupling between the thermoelectric and economic sub-problems. This Viewpoint article builds on the author's previous collaborative work [6,11], especially Ref. [6] which is the basis of the analytical expressions used here.

\section{The \$/W Equation}

We analyze the thermoelectric system using a typical lumped treatment with constant properties [6, 8, 9, 12] and assuming identical heat exchangers at the cold and hot sides, specified by their nominal area $A$ and heat transfer coefficient $U$. The legs have length $L$ and fill factor $F=A_{T E} / A$, where $A_{T E}$ is the sum of $n$ and $p$ leg areas [6]. The reservoirs are at fixed temperatures $T_{H}$ and $T_{C}$, while the junction temperatures are $T_{1}$ and $T_{2}$.

The $\$ / \mathrm{W}$ metric to be minimized is

$$
G=C / P .
$$

where $P$ is the electrical power generated and $C$ is the total capital cost of the module [13]. For simplicity the analysis ignores the costs of fuel, maintenance, salvage value, and interest rates, all of which could readily be incorporated into a more general analysis.

Although thermoelectric material costs have been most commonly expressed using units of $\$ / \mathrm{kg}$ [8-10] or $\$ / \mathrm{mol}$ [14], inspection of the textbook thermoelectric equations reminds us that module design is governed by lengths and areas, rather than mass or moles, and thus the most natural dimensions for representing material costs are volumetric $[6,8]$. We quantify these using $C^{\prime \prime \prime}$, with units of $\$ / \mathrm{cm}^{3}$. There are also manufacturing costs which scale with the area of a module $\left(C^{\prime \prime}: \$ / \mathrm{cm}^{2}\right)$, such as dicing and metallization, although a more detailed investigation $[1,6]$ showed that these are usually negligible for most practical materials and designs, and thus for simplicity we take $C^{\prime \prime}=0$ for the rest of this paper. Finally, and crucially, we include the costs of the heat exchanger and associated ceramic spreading plates ( $C_{H X} U,\left[\$ / \mathrm{cm}^{2}\right]$, here representing the sum of hot and cold sides [6]). Thus,

$$
C=\left(C^{\prime \prime \prime} L+C^{\prime \prime}\right) A F+C_{H X} U A \text {. }
$$

The electrical power is simply

$$
P=\eta_{T E} Q_{H}
$$

where $Q_{H}$ is the heat flow entering the thermoelectric module and $\eta_{T E}=\frac{\left(T_{1}-T_{2}\right)}{T_{1}} \frac{(\sqrt{1+Z T}-1)}{\sqrt{1+Z T}+T_{2} / T_{1}}$ is the efficiency of the thermoelectric generator.

The challenge is that the junction temperatures $T_{1}$ and $T_{2}$ are not pre-determined, but instead depend on the details of the thermoelectric design, including the free parameters $L$ and $F$. When costs are ignored, it is well known $[6,8,15]$ that $P$ is maximized when the thermal impedance of the thermoelectric module is close to double that of each heat exchanger, so that $L / F \approx 2 k / U$, where $k$ is the thermal conductivity, and thus $T_{1}$ and $T_{2}$ are approximately $\left(\frac{3}{4} T_{H}+\frac{1}{4} T_{C}\right)$ and $\left(\frac{1}{4} T_{H}+\frac{3}{4} T_{C}\right)$, respectively. The cost optimization below makes no presupposition about the suitability of such thermal impedance matching, although in the end it will turn out that $G$ minimization does correspond closely with $P$ maximization for most cases.

An analytical solution of Eqs. (1)-(3) was obtained in Ref. [6] subject to several approximations. Generalizing that development to remove the assumptions of small $Z T$ and small $T_{H}-T_{C}$, we find [16] 


$$
G \approx\left(\frac{\sqrt{1+Z T}+f(r) \cdot r^{-1}}{\sqrt{1+Z T}-1}\right)\left(\frac{T_{\text {avg }}\left(1+\frac{1}{4} \frac{\Delta T_{\text {res }}}{\text { Tovg }_{j}}\right)}{k \Delta T_{\text {res }}^{2}}\right)\left(\frac{2 F k}{U L}+1\right)^{2}\left(C^{\prime \prime \prime} L^{2}+C^{\prime \prime} L+\frac{C_{H X} U L}{F}\right)
$$

where $T_{\text {avg }}=\left(T_{H}+T_{C}\right) / 2, \Delta T_{r e s}=T_{H}-T_{C}, r=T_{H} / T_{C}$, and the correction factor $f(r)=\frac{3+r}{3+r^{-1}}$ is a weak function of $r$. The calculations of this paper use the reservoir parameters specified in Table I, which correspond to $r=\frac{7}{3}$ and $f(r)=1.556$.

\begin{tabular}{|c|c|c|c|c|c|c|}
\hline \multicolumn{4}{|c|}{ Reservoir and Heat Exchanger } & \multicolumn{3}{|c|}{ Material } \\
\hline $\begin{array}{l}\text { Hot Side } \\
\text { Reservoir } \\
\text { Temperature }\end{array}$ & $\begin{array}{l}\text { Cold Side } \\
\text { Reservoir } \\
\text { Temperature }\end{array}$ & $\begin{array}{l}\text { HX Heat } \\
\text { Transfer } \\
\text { Coefficient }\end{array}$ & $\begin{array}{l}\text { Cost }(\mathrm{HX}+ \\
\text { ceramic } \\
\text { plates })\end{array}$ & $\begin{array}{l}\text { Figure } \\
\text { of Merit }\end{array}$ & $\begin{array}{l}\text { Thermal } \\
\text { Conductivity }\end{array}$ & $\begin{array}{l}\text { Material } \\
\text { Cost }\end{array}$ \\
\hline $\mathrm{T}_{\mathrm{H}}=700 \mathrm{~K}$ & $\mathrm{~T}_{\mathrm{C}}=300 \mathrm{~K}$ & $U=100 \mathrm{~W} / \mathrm{m}^{2} \mathrm{~K}$ & $\begin{array}{l}C_{H X}=10 \$ / \\
(\mathrm{W} / \mathrm{K})\end{array}$ & $Z T=1$ & $\mathrm{k}=2 \mathrm{~W} / \mathrm{m}-\mathrm{K}$ & $\begin{array}{l}C^{\prime \prime}=1 \\
\$ / \mathrm{cm}^{3}\end{array}$ \\
\hline
\end{tabular}

Table I. Reference scenario. The reservoir and heat exchanger parameters are used throughout this paper, while these material parameters are used only in Fig. 2 and Table III.

\begin{tabular}{|c|c|c|c|c|c|c|c|}
\hline ID\# & Type & ZT & $C^{\prime \prime \prime}\left[\$ / \mathrm{cm}^{3}\right]$ & $\mathrm{C}_{\mathrm{M}}=\mathrm{C}^{\prime \prime \prime} / \rho[\$ / \mathrm{kg}]$ & $k[\mathrm{~W} / \mathrm{m}-\mathrm{K}]$ & $L_{20}[\mathrm{~mm}]$ & $F_{20}$ \\
\hline 2 & $\mathrm{Bi}_{2-x} \mathrm{Sb}_{x} \mathrm{Te}_{3}$ : Baseline bulk. & 1.05 & 0.86 & 125. & 1.36 & 3.0 & 0.077 \\
\hline 3 & $\mathrm{Bi}_{2-x} \mathrm{Sb}_{x} \mathrm{Te}_{3}:$ Nanobulk. & 1.52 & 0.88 & 137. & 0.67 & 2.2 & 0.10 \\
\hline 6 & $\mathrm{Bi}_{2-x} \mathrm{Sb}_{x} \mathrm{Te}_{3}$ : Epitaxial Superlattice. & 1.96 & 350. & 40,000 & 0.58 & 0.11 & 0.005 \\
\hline 31 & Polymer (PEDOT:Tos) & 0.25 & 0.0005 & 0.34 & 0.37 & 8.1 & 1.0 \\
\hline 1 & $\mathrm{Bi}_{2} \mathrm{Te}_{3}:$ Baseline bulk & 0.74 & 0.89 & 110. & 1.61 & 3.1 & 0.073 \\
\hline 5 & PbTe/Ag 2 Te: Nanostructured & 1.45 & 0.75 & 81. & 0.87 & 2.7 & 0.099 \\
\hline 7 & LAST $\left(\mathrm{AgPb}_{18} \mathrm{SbTe}_{20}\right)$ & 1.31 & 0.78 & 84. & 1.40 & 3.3 & 0.078 \\
\hline 12 & $\mathrm{Mg}_{2} \mathrm{Si}_{0.6} \mathrm{Sn}_{0.4}$ & 1.05 & 0.017 & 4.0 & 2.96 & 32. & 0.38 \\
\hline 27 & Half-Heusler ((Zr,Hf)NiSn) & 1.38 & 0.081 & 9.7 & 2.79 & 15. & 0.17 \\
\hline
\end{tabular}

Table II. Selected material highlights [1, 2], and $L_{20}$ and $F_{20}$ from Eq. (5).

\section{Minimum $L$ and $F$ : The Paradox of the Vanishing Leg}

As a reference scenario we consider the parameters summarized in Table I, with realistic heat exchanger values, and material properties corresponding loosely to mainstream bulk materials. Calculations for 31 real materials are presented later in this paper, using the detailed cost and property information for 30 materials from Ref. [1], as well as a recent high-ZT polymer (material ID\#31: like \#30 but with updated $k$ and ZT values from Ref. [2]). Selected material highlights are repeated in Table II for convenience.

We first consider $G$ optimization based only on the cost of the thermoelectric material and its associated manufacturing, which up until recently has been the conventional thinking. One approach [7-9] would begin with power maximization and $L / F \approx 2 k / U$. This calculation is depicted in Table III, for the five fixed values of $F$ specified in columns (a) to (e). For a heat exchanger reference area of $A=1 \mathrm{~m}^{2}$, all five designs take in 10,000 W of heat at the hot side and generate $664 \mathrm{~W}$ of electricity, for an efficiency of 6.6\%. Since the cost in Eq. (2) scales with $L$ and $F$, this immediately implies that the system cost can be driven to zero, simply by reducing $L$ and $F$ together $[6,9]$. But this strategy of miniaturization must eventually break down, because it seems absurd to imagine $L$ and $F$ both going truly to zero in a real device. We must address this paradox of the vanishing thermoelectric leg. 


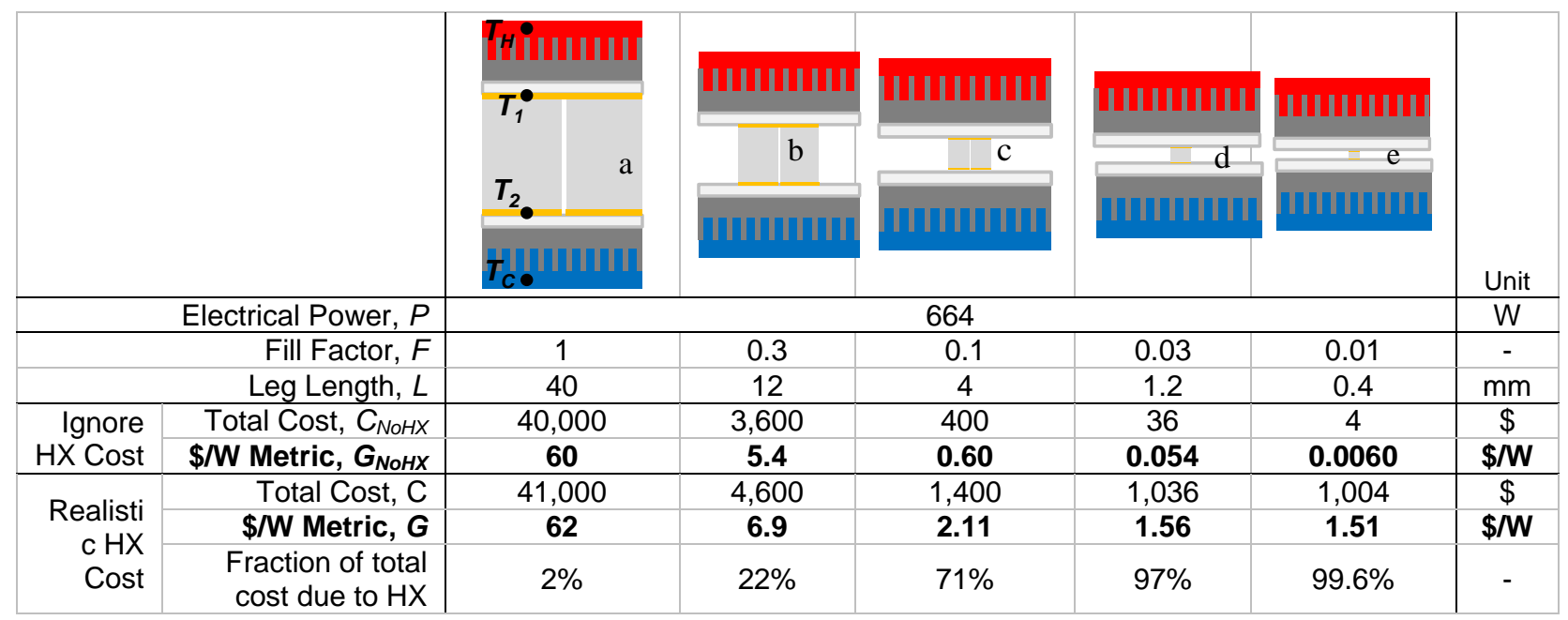

Table III. The paradox of the vanishing thermoelectric leg: a sample calculation using the scenario of Table I and a fixed $\mathrm{HX}$ reservoir area of $1 \mathrm{~m}^{2}$. Columns (a) through (e) correspond to reducing the fill factor and leg size together while still maintaining maximum power. If the costs of $\mathrm{HX}$ and ceramic plate are ignored, this progression from (a) to (e) leads to absurd conclusions like the system cost $G$ falling below $0.01 \$ / W$. On the other hand, accounting for realistic $\mathrm{HX}$ and plate costs gives a floor to the system cost, with little additional benefit in $G$ for reducing $F$ below 0.03 . These calculations use $C^{\prime \prime}=0$; accounting for a typical $C^{\prime \prime} \approx 0.02 \$ / \mathrm{cm}^{2}$ [1] has very little effect.

\section{Parasitics resolve the paradox}

In real devices the minimal values of $L$ and $F$ are limited by parasitic phenomena that are well known, but are commonly neglected [8, 9] including in the development of Eq. (4) [6]. Three of the most important parasitics are depicted in the $(L, F)$ regime map of Fig. 2(left).

First, $L_{\min }$ will be limited by electrical contact resistance $R_{c, e}[17,18]$. The objective is $L / \sigma>>2 R_{c, e}^{\prime \prime}$, which for typical values ( $R_{c, e}^{\prime \prime} \approx 10^{-6}-10^{-5} \Omega \mathrm{cm}^{2}, \sigma \approx 10^{3} \Omega^{-1} \mathrm{~cm}^{-1}$ ) suggests $L$ down to $\sim 0.1 \mathrm{~mm}$ should be viable. Indeed devices with $L=0.2 \mathrm{~mm}$ down to $0.04 \mathrm{~mm}$ are commercially available [19, 20]. A representative limit of $L_{\text {min }}=0.2 \mathrm{~mm}$ is depicted by the vertical line in Fig. 2 . The analogous thermal contact resistances are typically $R_{c, t h}^{\prime \prime} \approx 10^{-7} \mathrm{~m}^{2} \mathrm{~K} / \mathrm{W}$ and are negligible for even smaller values of $L$.

Aggressive reductions in $F_{\min }$ will be limited by the thermal spreading resistance in the ceramic plates that join the thermoelectric legs to the heat exchanger. NASA's highly-successful GPHS-RTG uses $F=0.034$ [21], and $F_{\min }$ down to 0.01 has been suggested as still practical using high thermal conductivity plates [8], which is the value depicted in Fig. 2.

$F_{\min }$ is also limited by the parallel heat flow through the open space between the plates and legs, leading to a restriction $\frac{L}{\mathrm{kFA}}<<\frac{2}{h_{\text {Shunt }}(1-F) A}$. For natural convection in air, $h_{\text {Shunt }}$ may easily be 5 or $10 \mathrm{~W} / \mathrm{m}^{2} \mathrm{~K}$ or even larger, which would begin to encroach on the (a) $\rightarrow$ (e) series of Table III; while for evacuated devices with metallized low-emissivity surfaces, the equivalent $h_{\text {Shunt }}$ for radiation could be $1 \mathrm{~W} / \mathrm{m}^{2} \mathrm{~K}$ or smaller (diagonal line in Fig. 2). 

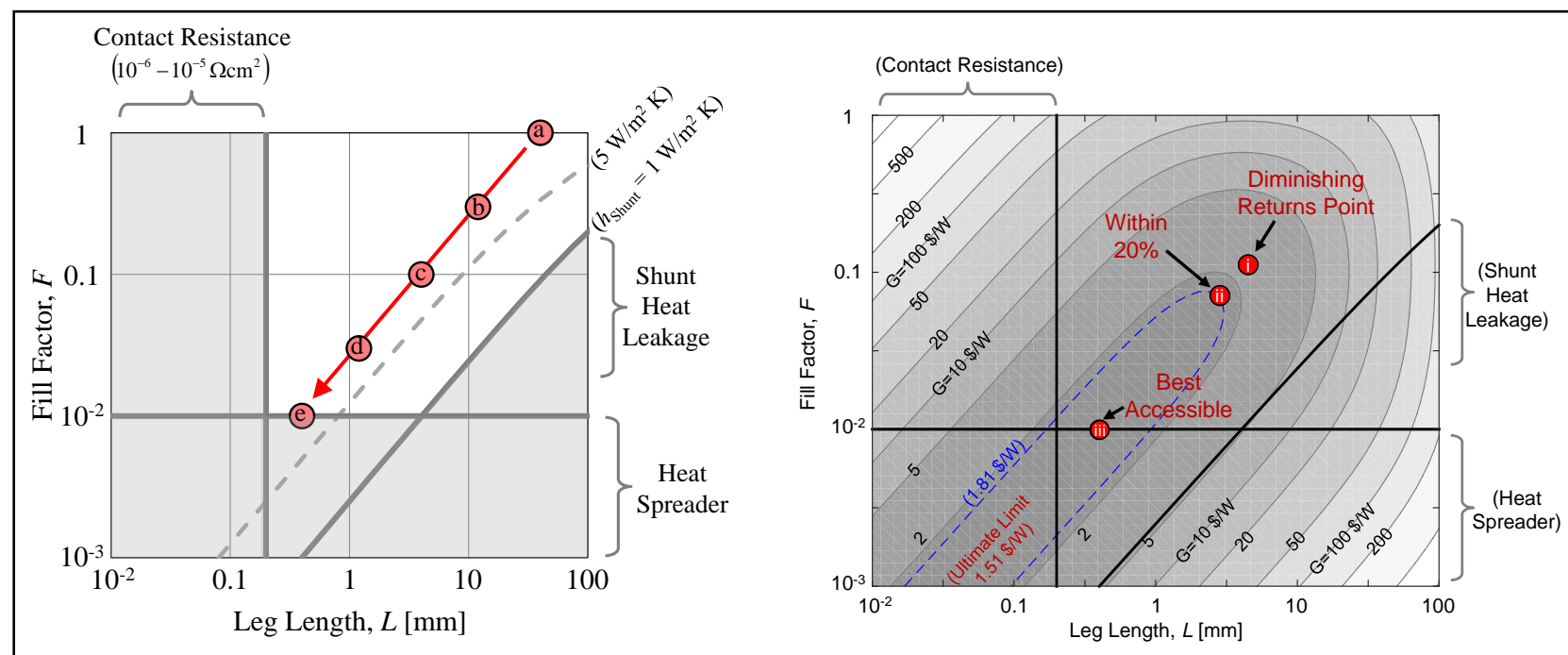

Fig. 2. Left: Representative calculations for three parasitic phenomena that limit $L$ and $F$ in practical devices. Overlaid are points representing designs (a)-(e) from Table III. This example shows how devices may remain practical down to $F \approx 0.01$ and $L \approx 0.2 \mathrm{~mm}$. Right: Contours of the $\$ / W$ system cost, $G$, for the scenario of Table I including both material and $\mathrm{HX}$ cost. The lowest cost region is the dark trough, with $G \rightarrow 1.51 \$ / \mathrm{W}$ for vanishing $L$ and $F$. Given the practical constraints on $L$ and $F$ (black lines), the best accessible design is indicated by point (iii), and gives a $G$ value within $1 \%$ of the ultimate limit. Also shown is the "good enough" contour (dashed blue line), indicating designs with $G$ within $20 \%$ of the ultimate limit. Point (ii) is the target $20 \%$ point which is used in Fig. 3 to define targets for all 31 materials. (Point (i) indicates a characteristic "diminishing returns point" defined previously in Ref. [6] but not used here.)

\section{Practical Cost-0ptimization of $L$ and $F$ for 31 Materials}

Referring back to the five designs presented in Table III, Fig. 2(left) has verified that designs (a) - (d) are consistent with practical realities, and even design (e) may be borderline. The smallest leg geometry compatible with the imposed restrictions is Point (iii) in Fig. 2(right), with $L=0.40 \mathrm{~mm}$ and $F=0.010$. Here the system cost is within $1 \%$ of the ultimate limit of $1.51 \$ / \mathrm{W}$; that is, over $99 \%$ of the system capital expenditure would be on the heat exchanger and ceramic plates, not the thermoelectric material itself. Furthermore, nearly the same performance can be achieved even with substantially larger legs: at point (ii) ( $L=L_{20}=2.8 \mathrm{~mm}, F=F_{20}=0.071$ ), the system cost of $1.81 \$ / \mathrm{W}$ is still within $20 \%$ of the ultimate limit. We use this $20 \%$ threshold to define a "good enough" design target $\left(L_{20}, F_{20}\right)$, at which point the material cost is relatively unimportant (only $1 / 5$ the cost of the heat exchanger and plates) for the overall system.

This important conclusion turns out to be quite general and should be achievable for all but the most costly materials. To see this, we consider all 31 materials from Refs. [1, 2] and calculate the characteristic design targets $\left(L_{20}, F_{20}\right)$ following Ref. [6] as

$$
L_{20}=\sqrt{\frac{2 C_{H X} k_{\text {eff }}}{5 C^{\prime \prime \prime}}} \text { and } F_{20}=\sqrt{\frac{U^{2} C_{H X}}{10 k_{\text {eff }} C^{\prime \prime \prime}}} \text {. }
$$

For slightly improved accuracy [6] we have invoked an effective thermal conductivity,

$$
k_{\text {eff }} \approx k(1+0.4 Z T)
$$

which is a convenient linearization [6, 22] of a helpful result first obtained by Baranowski, Snyder, and Toberer [23].

The resulting $\left(L_{20}, F_{20}\right)$ for all 31 materials are given in Fig. 3. Nearly all materials have their $\left(L_{20}, F_{20}\right)$ targets well within the zone of practicality. Note especially the cluster of high-ZT materials around 


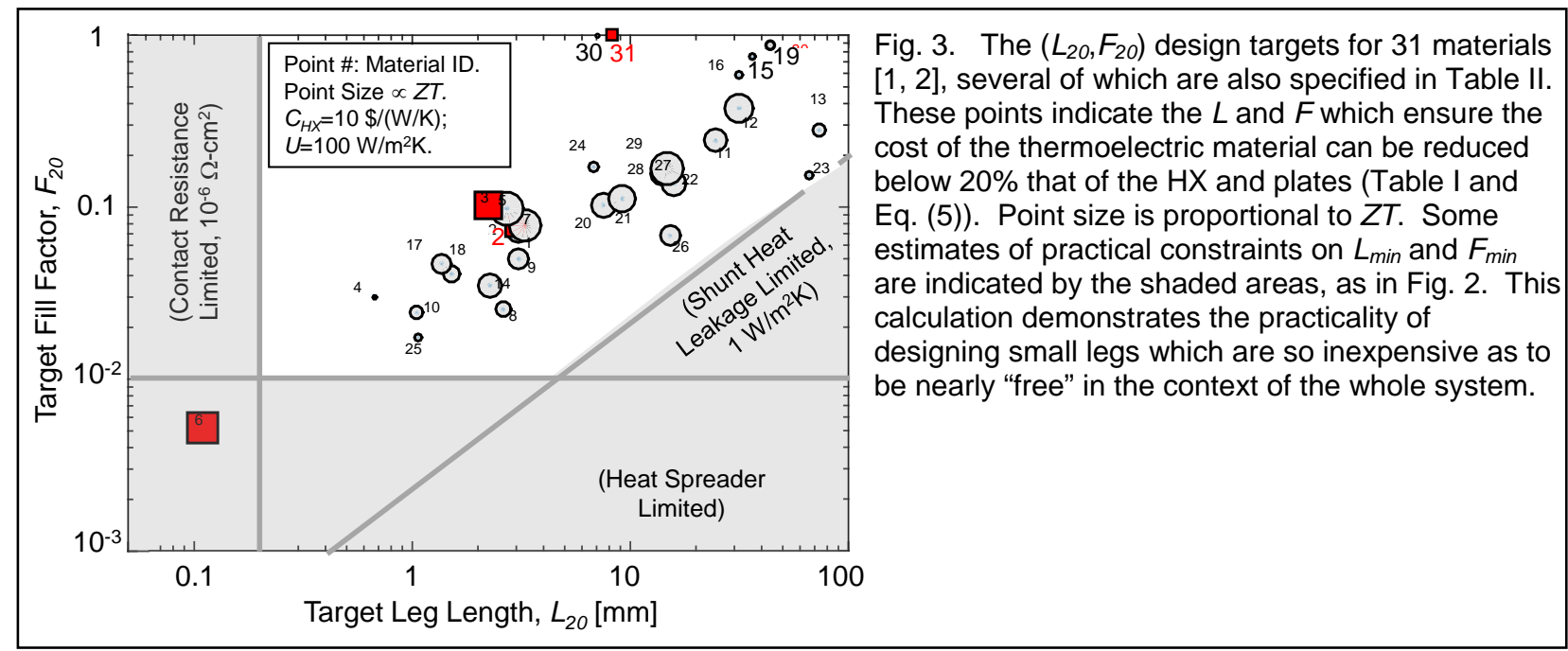

$\left(L_{20}=2 \mathrm{~mm}-3 \mathrm{~mm}, F_{20}=0.07-0.10\right)$, many of which are specified in Table II. This cluster includes a benchmark bulk $\mathrm{Bi}_{2} \mathrm{Te}_{3}$ (ID\#1), nanostructured PbTe/Ag $2 \mathrm{Te}$ (ID\#5, Ref. [24]), and LAST (ID\#7, $\mathrm{AgPb}_{18} \mathrm{SbTe}_{20}$, Ref. [25]). Also notable later will be a half-Heusler (ID \#27, (Zr,Hf)NiSn, Ref. [26]). This leads to the very important conclusion that in real devices, for nearly all materials of contemporary interest, it should be possible to reduce $L$ and $F$ to the point where the thermoelectric material cost is negligible, and instead the system cost is dominated by the ceramic plates and heat exchanger. The lone exception in Fig. 3 is Material \#6, which represents an epitaxial superlattice with an extremely expensive $C^{\prime \prime \prime} \approx 350 \$ / \mathrm{cm}^{3} \approx 40,000 \$ / \mathrm{kg}$.

\section{Completing the Story: Figure 1 Revisited}

Having seen the strong motivation for reducing $F$ and $L$, as well as their practical limitations, we can now make Fig. 1 quantitative. We again use the reservoir and heat exchanger conditions of Table I, and now impose $L \geq 0.5 \mathrm{~mm}, F \geq 0.05$ which are slightly more conservative (larger legs) than the shaded bounds used above in Figs. 2 and 3.

The full analysis [6] shows that the surface $G\left(Z T, C^{\prime \prime \prime}\right)$ is not truly universal because it also depends on each material's thermal conductivity. In order to visualize the results for all 31 materials on a single plot, we must choose a single representative value of $k$. We choose $k=2 \mathrm{~W} / \mathrm{m}-\mathrm{K}$ which lies in the middle of the range reported for nearly all of the interesting high-ZT materials from Ref. [1]. As a check, the calculations were repeated using each material's proper $k$, which verified that this only impacts the corresponding optimal $G$ values at the level of 10 s of percent, without changing the qualitative ranking of the materials or the main conclusions below.

The final results for all 31 materials are presented in Fig. 4. Just as in Fig. 1, the contours represent constant system cost, with the best results towards the bottom right (darker shading: smaller $G$ ). After optimizing $L$ and $F$ subject to the aforementioned constraints, Fig. 4 shows that this set of materials spans a very large range of attainable system costs. The best materials are ID\# 3, 5, 7, and 27, and can achieve $G$ between 1.2 and $1.4 \$ / \mathrm{W}$, suggesting the potential to compete with geothermal and concentrating solar power, and possibly even coal and natural gas [1]. For the most part the lowest $G$ solutions are found in the materials with the highest ZT (largest size points), regardless of the materials' cost. The dramatic exception is material \#6, the epitaxial superlattice with the highest considered ZT. Even after using the smallest allowed $F$ and $L$, the cost of the leg is still far too high, and its optimized system $G$ of $15 \$ / \mathrm{W}$ is an order of magnitude higher than the other best materials. 


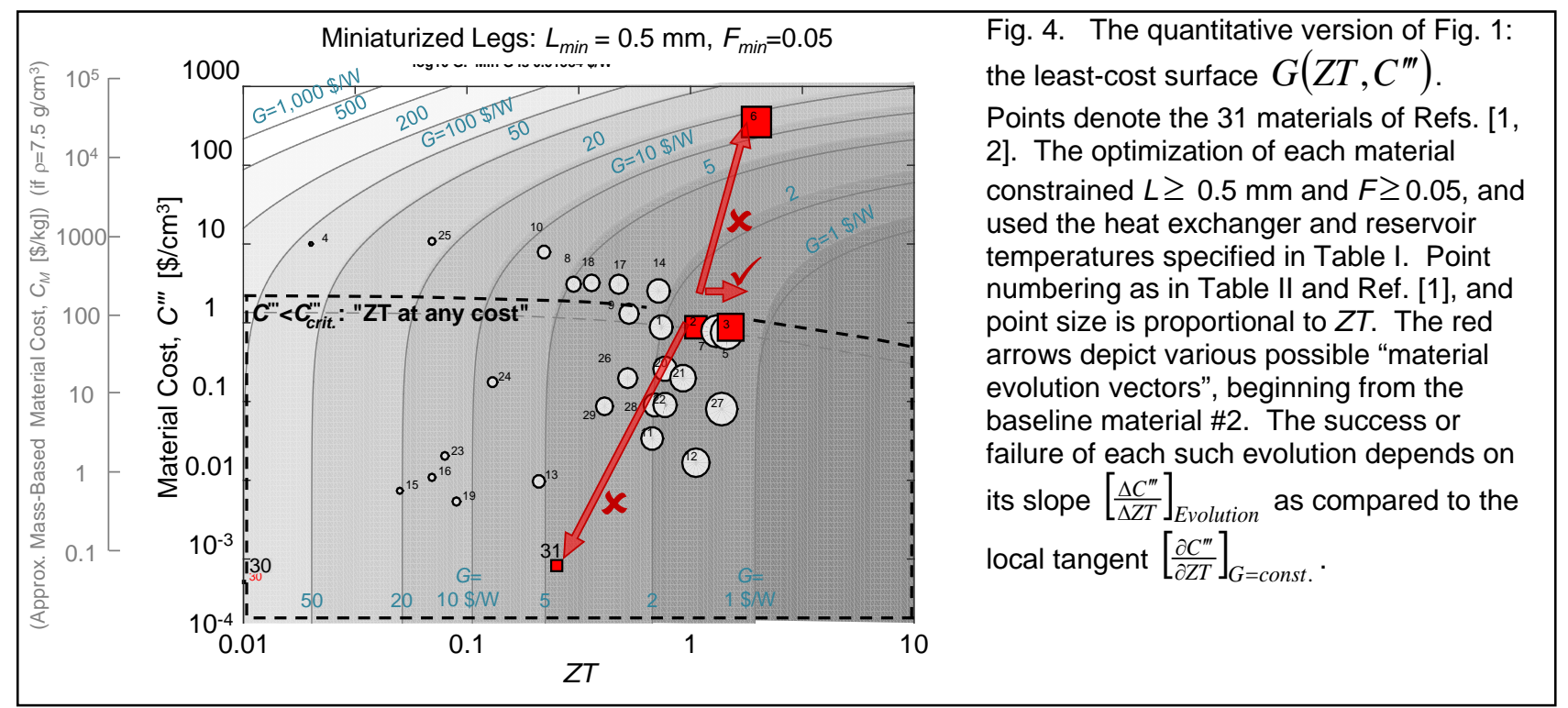

With Fig. 4 we can now revisit quantitatively the three key issues which were raised qualitatively in the Introduction around Fig. 1:

\section{The nanostructuring approach: Paying a premium for higher ZT}

Consider the three materials of the $\mathrm{Bi}_{2-\mathrm{x}} \mathrm{Sb}_{\mathrm{x}} \mathrm{Te}_{3}$ family (\#2, \#3, and \#6) in Fig. 4. Material \#2 is a baseline bulk material, and can achieve a system cost of $1.58 \$ / \mathrm{W}$. This material is also available in two nanostructured forms: an epitaxial superlattice (\#6) and bulk nanocrystal (\#3), both of which offer higher $Z T$ at a price premium as compared to \#2. However, the calculation in Fig. 4 shows that the $Z T$ gain in the superlattice material is far too small to justify the more than 300 -fold increase in material price, and so the system metric is actually far worse, at $15 \$ / \mathrm{W}$. On the other hand, material \#3 is only slightly more expensive than \#2 but increases $Z T$ by around $50 \%$, leading to an overall reduction in $G$ down to 1.25 \$/W.

It is instructive to compare the slopes of the "material evolution vectors" $(2 \rightarrow 3)$ and $(2 \rightarrow 6)$ indicated by red arrows in Fig. 4. We see that developing premium materials with higher $Z T$ is a viable strategy only if the slope of $\left[\frac{\Delta C^{\prime \prime}}{\Delta Z T}\right]_{\text {Material Evolution }}$ is gentle enough. This slope test is also depicted in Fig. 1, and will be made quantitative shortly below.

\section{The polymer approach: Sacrificing ZT to use much cheaper materials}

In Fig. 4 the representative polymers are \#30 (PEDOT:PSS [27]) and \#31 (PEDOT:Tos [2]). As compared to the baseline bulk $\mathrm{Bi}_{2-\mathrm{x}} \mathrm{Sb}_{\mathrm{x}} \mathrm{Te}_{3}$ (\#2), the best polymer considered here (\#31) has a $Z T$ that is $4 \mathrm{x}$ smaller, but with a cost savings of around 1,700x (volumetric basis). Only after referring to the calculations in Fig. 4 can we see which effect is more important: unfortunately, the ZT penalty still overwhelms even the dramatic cost savings, and the optimized system cost of $4.54 \$ / \mathrm{W}$ is still 2.9x higher than that from the reference bulk material. Graphically, this polymer approach was unsuccessful because the evolution vector $(2 \rightarrow 31)$ has a slope $\left[\frac{\Delta C^{\prime \prime \prime}}{\Delta Z T}\right]_{\text {Material Evolution }}$ that is too gentle. See also the two arrows for the polymer approach in Fig. 1.

\section{Graphical interpretation: A slope test}

A key graphical interpretation of Fig. 1 is that the tradeoff between $Z T$ and material cost is represented by the slope of the curve $C^{\prime \prime \prime}=f(Z T)$ for a fixed value of $G$. Consider a materials development strategy 
that will evolve the properties of a material from some baseline coordinates $\left(C^{\prime \prime \prime}, Z T\right)_{\text {Baseline }}$. The strategy succeeds if its evolution vector $\left(\Delta C^{\prime \prime \prime}, \Delta Z T\right)_{\text {Evolution }}$, depicted as a red arrow, ends up in a region of $G<G_{\text {Baseline }}$. From standard concepts of vector calculus, this is equivalent to the following slope test. If the material evolution is based on increasing both $C^{\prime \prime \prime}$ and $Z T$ (the "nanostructuring approach"), it succeeds when $\left[\frac{\Delta C^{\prime \prime \prime}}{\Delta Z T}\right]_{\text {Nanostructuring }}<\left[\frac{\partial C^{\prime \prime \prime}}{\partial Z T}\right]_{G=G_{\text {Baseline }}}$. On the other hand, if the evolution involves reducing both $C^{\prime \prime \prime}$ and ZT (the "polymer approach"), referring to Fig. 1 it is also easy to see that the slope test changes sign, and success now requires $\left[\frac{\Delta C^{\prime \prime \prime}}{\Delta Z T}\right]_{\text {PolymerApproach }}>\left[\frac{\partial C^{\prime \prime \prime}}{\partial Z T}\right]_{G=G_{\text {Baseline }}}$.

\section{The limit of free material: $C_{c r i t .}^{\prime \prime \prime}$ and the region of "ZT at any cost"}

In the calculations of Fig. 4, the material costs of the polymers (\#30 and \#31) are already so low that they might as well be free. Even increasing the polymer's $C^{\prime \prime \prime}$ a thousand-fold would have almost no impact on the resulting system $G$. Likewise, for sufficiently small $C^{\prime \prime \prime}$, the slope $\left[\frac{\partial C^{\prime \prime \prime}}{\partial Z T}\right]_{G=\text { const. }}$ becomes arbitrarily steep, because once the material is cheap enough it no longer influences $G$, in which case materials evolution favors increasing $Z T$ at any cost.

To quantify this region of virtually free material, we introduce a dimensionless sensitivity [28],

$$
S=\frac{Z T}{C^{\prime \prime}}\left[\frac{\partial C^{\prime \prime \prime}}{\partial Z T}\right]_{G}=\left[\frac{\partial \ln C^{\prime \prime \prime}}{\partial \ln Z T}\right]_{G} \text {. }
$$

$S$ is the power law exponent in a local curve fit $C^{\prime \prime \prime} \propto(Z T)^{S}$ for a constant-G line. This means $S$ is also exactly the slope in the log-log plots of Figs. 1 and 4, which we have seen is so important to their graphical interpretation. Here we arbitrarily choose $S=10$ to define the threshold for " $Z T$ at any cost," whereby $G$ is 10 times more sensitive to $Z T$ than to $C^{\prime \prime \prime}$. At $S=10$, a small increase in $Z T$ by as little as $7.2 \%$ is still beneficial for $G$ even if the materials cost is doubled.

As indicated by the black dashed line, for the systems considered in Fig. 4 the large majority of presentday materials fall within the regime of "ZT at (nearly) any cost," with the most dramatic exception being the epitaxial superlattice (\#6). These observations about materials suitability are entirely consistent with Fig. 3: as long as the $L_{20}$ and $F_{20}$ targets are deemed practical, then the legs can be made sufficiently small such that the overall system cost is dominated by the heat exchanger and ceramic plates, rather than the legs themselves.

The threshold $C_{c r i t}^{\prime \prime \prime}$ depends strongly on the heat exchanger parameters as well as prescribed $F_{\min }$ and $L_{\min }$ which constrain the overall optimization. Figure 4 shows that the $Z T$ dependence of $C_{c r i t}^{\prime \prime \prime}$ is quite modest for practical $Z T$ values, since the dashed black line is approximately flat for $Z T<3$. Therefore we are justified in using a small-ZT approximation to estimate $C_{c r i t .}^{\prime \prime \prime}$. Applying Eq. (7) to results from Ref. [6] gives $C_{\text {crit. }}^{\prime \prime \prime}=C_{H X} U / F L(S-1)$. For all 31 materials considered above, the optimizations were found to be limited by the prescribed $F_{\min }$ rather than $L_{\min }$, and the optimal $L$ settled approximately to the thermal impedance matched value of $L_{\text {opt }} \approx 2 k F_{\text {min }} / U$. Thus,

$$
C_{\text {crit. }}^{\prime \prime \prime} \approx \frac{C_{H X} U^{2}}{2 F_{\text {min }}^{2} k(S-1)} .
$$

Importantly, this threshold is independent of the reservoir temperatures. Equation (8) confirms that the $C_{c r i t .}^{\prime \prime \prime}$ value in Fig. 4 is around $1.1 \$ / \mathrm{cm}^{3}$. Using a typical density of $\rho \approx 7.5 \mathrm{~g} / \mathrm{cm}^{3}$ (roughly representative of many higher-ZT materials [1]), the equivalent mass-based cost is $C_{M, \text { crit. }} \approx 150 \$ / \mathrm{kg}$. Even materials 


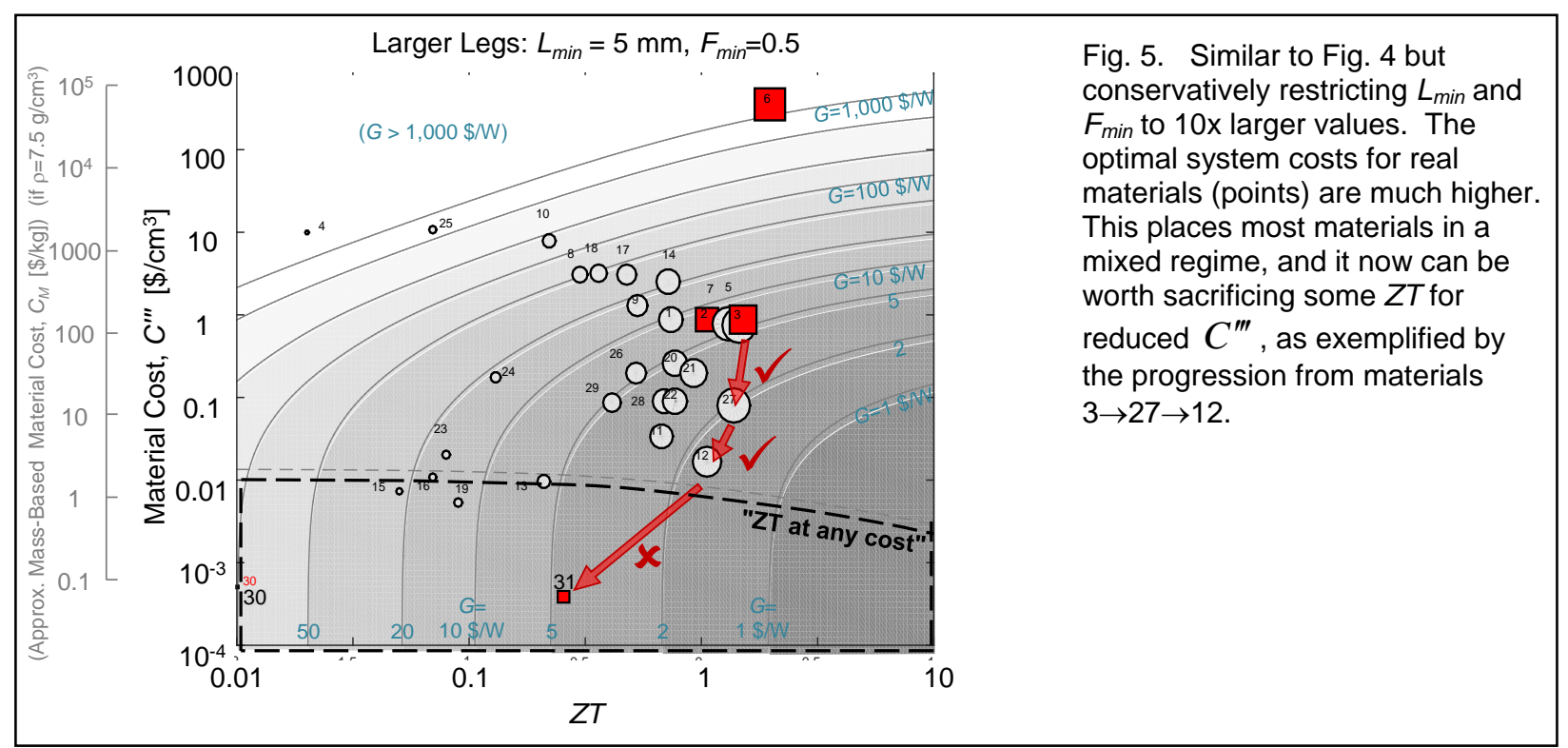

costing $1,000 \$ / \mathrm{kg}$ have $S \approx 2.3$, meaning the system cost is still more than twice as sensitive to $Z T$ than to $C^{\prime \prime \prime}$.

\section{The Other Limit: When Material Costs Dominate}

\section{Larger legs result in a much more costly system (Fig. 5)}

Figure 5 shows the same cost optimization using much more conservative constraints of $L_{\text {min }}=5 \mathrm{~mm}$ and $F_{\min }=0.5$, which would be closer to many traditional thermoelectric modules. Because the minimal leg volume is 100-fold larger than in Fig. 4, this calculation places much greater emphasis on the material cost.

The region of "ZT at any cost" is shifted downwards 100 -fold, to a threshold $C_{\text {crit. }}^{\prime \prime \prime}=0.011 \$ / \mathrm{cm}^{3}(\approx 1.5$ $\$ / \mathrm{kg}$ ). Since most materials are at least this costly, the tradeoff between $Z T$ and materials cost is more relevant, and the "polymer approach" depicted in Fig. 1 can now be fruitful. Consider the sequence from material $3 \rightarrow 27 \rightarrow 12 \rightarrow 31$, which in each step involves sacrificing $Z T$ to save on materials costs. The optimal $(L, F)$ design for Material \#3 gives $G_{\min }=5.7$ \$/W. Material \#27 has a slightly lower ZT (by 9\%) but 10 -fold lower material cost, with the combined effect of reducing $G$ to $1.9 \$ / \mathrm{W}$. This trend continues when evolving to Material 12, with the net result of slight further improvement in $G$ to $1.7 \$ / W$.

However, the vector from $\# 12 \rightarrow \# 31$ has a flatter slope $\left[\frac{\Delta C^{\prime \prime \prime}}{\Delta Z T}\right]_{\text {Evolution }}$, and furthermore brings the materials properties deep within the realm of " $Z T$ at any cost." Thus the additional reduction in material costs for the polymer have minimal benefit, and instead due to the small $Z T$ the system $G$ increases nearly three-fold, back up to 4.6 \$/W.

When materials costs matter, the figure of merit involves $\mathrm{ZT} /\left(\$ / \mathrm{cm}^{3}\right)$, not $\mathrm{ZT} /(\$ / \mathrm{kg})$ Consider the regions of Figs. 4 and 5 where $C^{\prime \prime \prime}>>C_{c r i t .}^{\prime \prime \prime}$. Referring to Eq. (8), this is expected if the material is very expensive (e.g. epitaxial superlattices), $F_{\min }$ is large, and/or the heat exchanger and ceramic plates are very cheap. The legs again should be miniaturized as much as possible. For the 31 materials considered above we find that the practical constraints on $F_{\min }$ are more restrictive than those on $L_{\text {min }}$, and this regime was considered previously in Ref. [6] (Eq. 41). The optimized system cost becomes 
$G \propto \frac{C^{\prime \prime} k F_{\min }^{2}}{Z T U^{2}}$ in the small $Z T$ limit, in which case materials should be ranked by the following costperformance figure of merit,

$$
\frac{Z T}{C^{\prime \prime} k} \text {. }
$$

This confirms that, when materials costs do matter, they enter most fundamentally on a volumetric basis $\left[Z T /\left(\$ / \mathrm{cm}^{3}\right)\right]$, rather than mass $[Z T /(\$ / \mathrm{kg})]$ or molar $[Z T /(\$ / \mathrm{mol})]$. Two subtleties must also be noted. For materials with $Z T \sim 1$ and above, the $Z T$-dependence is weaker than indicated in Eq. (9), thereby placing relatively more importance on the cost term. Second, $k$ appears in the denominator of Eq. (9) because the system was limited by a prescribed $F_{\min }$; if instead it were limited by the practicality of $L_{\min }$, the analogous figure of merit is $\frac{Z T k}{C^{\prime \prime}}$.

\section{Conclusion: Miniaturize the legs, and maximize ZT at (almost)} any cost

This Viewpoint article analyzed the tradeoff between $Z T$ and material cost when minimizing the \$/W metric $G$ for thermoelectric energy scavenging. Fuel costs were ignored, but would place even greater emphasis on ZT maximization. The key features of this tradeoff are presented in Figs. 1 (qualitatively) and 4 (quantitatively). The main conclusions are:

- To minimize the system cost, there is initially a strong incentive to shrink the thermoelectric legs' length and fill-factor. However, there is a point of diminishing returns, and eventually the system cost is dominated by cost of the heat exchanger and heat spreading plates (Table III and Fig. 2(right)).

- Thus there is a threshold material cost $C_{\text {crit. }}^{\prime \prime \prime}$ below which the material might as well be free (Figs. 1, 4, and 5). Figure 3 suggests that it should indeed be practical to reach this regime for realistic materials, heat exchangers, and parasitics, since for almost all materials considered the targets are in the range $1 \mathrm{~mm}$ $<L_{20}<10 \mathrm{~mm}$ and $0.03<F_{20}<0.3$.

- In this case, the goal for materials development is to maximize ZT at nearly any cost. Materials costs up to $C_{\text {crit. }}^{\prime \prime \prime} \sim 1 \$ / \mathrm{cm}^{3}(\sim 150 \$ / \mathrm{kg})$ might as well be free. Even at $C^{\prime \prime \prime} \sim 10 \$ / \mathrm{cm}^{3}(\sim 1,000 \$ / \mathrm{kg})$ the system cost is still more than twice as sensitive to changes in $Z T$ than $C^{\prime \prime \prime}$. Importantly, Fig. 4 shows that nearly all materials of present interest have $C^{\prime \prime \prime}$ comparable to or smaller than $C_{c r i t}^{\prime \prime \prime}$, the main exception being epitaxial nanomaterials.

- If for some reason leg miniaturization is deemed unacceptable, then rather than Fig. 4 it is Fig. 5 which is most relevant. In this case the tradeoff between $\mathrm{ZT}$ and material cost becomes important in practice, as exemplified by Eq. (9) and the progression from materials numbers $3 \rightarrow 27 \rightarrow 12$ in Fig. 5 .

- The most important assumptions of this work are the particular values chosen for the heat exchanger coefficient $U$ and its cost $C_{H X}$. These have a strong effect on the numerical targets for the miniaturized legs ( $L_{20}, F_{20}$ in Fig. 3) and in defining the boundary of the " $Z T$ at any cost” region ( $C_{\text {crit. }}^{\prime \prime \prime}$ in Figs. 1,4 , and 5). The effects of alternate choices for $U$ and $C_{H X}$ are easily explored by rescaling with Eqs. (5) and (8). 


\section{Acknowledgements}

The author thanks G. J. Snyder and G. Wehmeyer for helpful comments. This work was supported in part by the NSF (grant \#1055317).

\section{References}

[1] S. LeBlanc, S.K. Yee, M.L. Scullin, C. Dames, K.E. Goodson, Renewable \& Sustainable Energy Reviews, 32 (2014) 313-327.

[2] O. Bubnova, Z.U. Khan, A. Malti, S. Braun, M. Fahlman, M. Berggren, X. Crispin, Nat Mater, 10 (2011) 429-433.

[3] K. Branker, M.J.M. Pathak, J.M. Pearce, Renewable and Sustainable Energy Reviews, 15 (2011) 4470-4482.

[4] M.V. Vedernikov, E.K. Iordanishvili, in: Thermoelectrics, 1998. Proceedings ICT 98. XVII International Conference on, 1998, pp. 37-42.

[5] E. Altenkirch, Physikalische Zeitschrift, 10 (1909) 560-568.

[6] S.K. Yee, S. LeBlanc, K.E. Goodson, C. Dames, Energy \& Environmental Science, 6 (2013) 25612571.

[7] D.M. Rowe, G. Min, Iee Proceedings-Science Measurement and Technology, 143 (1996) 351-356.

[8] K. Yazawa, A. Shakouri, Environmental Science \& Technology, 45 (2011) 7548-7553.

[9] K. Yazawa, A. Shakouri, Journal of Electronic Materials, 41 (2012) 1845-1850.

[10] N.R. Kristiansen, G.J. Snyder, H.K. Nielsen, L. Rosendahl, Journal of Electronic Materials, 41 (2012) 1024-1029.

[11] C. Dames, S.K. Yee, S. LeBlanc, M.L. Scullin, K.E. Goodson, a.o.a.h.w.m.o.f.-. \$ per Watt Cost Metrics for Thermoelectric Power Generation: Beyond ZT. (Presentation CC3.02 at the Fall 2014 MRS Meeting, in, 2014.

[12] S.W. Angrist, Direct Energy Conversion, Allyn and Bacon, Inc., Boston, 1965.

[13] The currency should also be specified with respect to a reference year, for example, US dollars inflation-adjusted to 2013. This facilitates the straightforward comparison of different works, for example, with a conversion factor 2.2 [USD(2013)]/[GBP(1996)] to compare Refs. [1] and [7]. [14] G.G. Yadav, J.A. Susoreny, G.Q. Zhang, H.R. Yang, Y. Wu, Nanoscale, 3 (2011) 3555-3562.

[15] P.M. Mayer, R.J. Ram, Nanoscale and Microscale Thermophysical Engineering, 10 (2006) 143-155. [16] In relating T1 and T2 to TH and TC, this equation still makes the assumption of approximately equal heat flows through both heat exchangers, introducing relative errors of order $n \_T E[6]$.

[17] H. Bottner, J. Nurnus, A. Schubert, Chapter 46: Miniaturized Thermoelectric Converters, in: D. Rowe (Ed.) Thermoelectrics Handbook: Macro to Nano, CRC Press, 2006.

[18] J.P. Fleurial, G.J. Snyder, J.A. Herman, P.H. Giauque, W.M. Phillips, M.A. Ryan, P. Shakkottai, E.A. Kolawa, M.A. Nicolet, Eighteenth International Conference on Thermoelectrics. Proceedings, ICT'99 (Cat. No.99TH8407), (1999) 294-300.

[19] V. Semenyuk, Journal of Electronic Materials, 43 (2014) 1539-1547.

[20] H. Bottner, J. Nurnus, Ch. 17 Miniaturized Thermoelectric Converters, Technologies, and Applications, in: D.M. Rowe (Ed.) Thermoelectrics And Its Energy Harvesting, Volume 2: Modules, Systems, And Applications In Thermoelectrics, Taylor \& Francis, 2012.

[21] G.L. Bennett, J.J. Lombardo, R.J. Hemler, G. Silverman, C.W. Whitmore, W.R. Amos, E.W. Johnson, A. Schock, R.W. Zocher, T.K. Keenan, J.C. Hagan, R.W. Englehart, in: 4th International Energy Conversion Engineering Conference and Exhibit (IECEC), Paper AIAA 2006-4096, American Institute of Aeronautics and Astronautics, San Diego, CA, 2006. 
[22] S.K. Yee, S. LeBlanc, K.E. Goodson, C. Dames, Energy \& Environmental Science, 7 (2014) 34413442.

[23] L.L. Baranowski, G. Jeffrey Snyder, E.S. Toberer, Journal of Applied Physics, 113 (2013) -.

[24] Y. Pei, N.A. Heinz, A. LaLonde, G.J. Snyder, Energy \& Environmental Science, 4 (2011) 3640-

3645.

[25] K.F. Hsu, S. Loo, F. Guo, W. Chen, J.S. Dyck, C. Uher, T. Hogan, E.K. Polychroniadis, M.G.

Kanatzidis, Science, 303 (2004) 818-821.

[26] S. Sakurada, N. Shutoh, Applied Physics Letters, 86 (2005) 082105.

[27] M. Scholdt, H. Do, J. Lang, A. Gall, A. Colsmann, U. Lemmer, J. Koenig, M. Winkler, H. Boettner, Journal of Electronic Materials, 39 (2010) 1589-1592.

[28] B.C. Gundrum, D.G. Cahill, R.S. Averback, Physical Review B, 72 (2005) 5. 